signs and symptoms of RE. MRI abnormalities had a focal onset and spread across one hemisphere with late progressive atrophy. The earliest abnormal MRI finding was cortical swelling with hyperintense T2/FLAIR signal. Histopathology showed a higher number of $T$ cells and reactive astrocytes in the earlier MRI focal stages compared to the late atrophic stage. The early stages are characterized by the highest inflammatory changes and late stages are mainly atrophic. (Bien CG, Urbach H, Deckert M et al. Diagnosis and staging of Rasmussen's encephalitis by serial MRI and histopathology. Neurology January (2 of 2) 2002;58:250-257). (Reprints: Dr C Bien, University of Bonn, Department of Epileptology, Sigmund-Freud-Str 25, D-53105 Bonn, Germany).

COMMENT. The course of RE is characterized by an early inflammatory stage, as judged by density of $T$ cells and microglial nodules, followed by a decrease in inflammation and an atrophic stage. These inflammatory changes have a temporal or frontocentral onset and spread across a hemisphere, as judged by MRI. Deterioration with atrophy occurs within the first 2 years after onset. If a diagnostic brain biopsy is pianned, early inflammatory changes should be found in areas of increased MRI signal. The data suggest that immunosuppressive treatment may be most effective during stages of active inflammation, usually in the first 15 months after onset. Hemispherectomy is usually reserved for patients with a residual hemiplegia, at the late atrophic stage.

\title{
SPHENOID SINUSITIS AND MIGRAINE-TYPE HEADACHE
}

Three case histories of children (ages 10, 12, and 14 years) with isolated sphenoid sinusitis who presented with acute, subacute, and chronic headache symptoms resembling migraine are reported from the University of TexasHouston Medical School. The acute headaches (patient 1) were associated with vomiting, insomnia, photophobia, and phonophobia. Subcutaneous sumatriptan 6 mg resulted in only modest relief of symptoms. CT showed sphenoid sinus opacification. Symptoms resolved with antibiotics. The subacute headaches (patient 2) were bifrontal and throbbing, and occurred $30 \mathrm{~min}$ after awakening. The headaches were associated with photophobia and phonophobia, and they occurred almost daily. Mother had a history of migraine. CT scan showed opacification of sphenoid sinuses. Headaches resolved following antibiotic therapy. Patient 3 was referred with a diagnosis of migraine. He had a 3 year history of spenoid sinusitis, previously treated with surgical drainage, and frequent retro-orbital headaches associated with photophobia. Family history was strongly positive for migraine. CT showed opacity of the left sphenoid sinus. He was treated with propranolol and endoscopy with surgical drainage, followed by weekly injections for mold allergy. Headaches were relieved and propanolol was discontinued. Facial tenderness was absent in all cases, and nasal congestion occurred only in patient 3, with chronic headaches. (Ng Y, Butler IJ. Sphenoid sinusitis masquerading as migraine headaches in children. I Child Neurol Dec 2001;16:882-884). (Respond: Dr Ian J Butler, Department of Neurology, University of TexasHouston Medical School, 6431 Fannin, Room 7.044, Houston, TX 77030).

COMMENT. Isolated sphenoid sinusitis in children may present with headaches resembling migraine. The typical symptoms of sinusitis, with nasopurulent discharge and fever, are frequently absent. The proximity of the sphenoid sinus to the cavernous sinus, and the course of the cranial nerves III, IV, V, and VI, adds to the potential for serious complications. The most common causes are an upper respiratory tract infection and allergies. Branches of the trigeminal nerve, ophthalmic and maxillary divisions, supply the sensory 
innervation of the sphenoid sinus. This explains the similarities of the sphenoid sinus headache and migraine headache, both involving a trigeminovascular mechanism. A high index of suspicion is required in diagnosis. Sometimes the diagnosis is made incidentally, following an MRI obtained because of chronic headaches. In acute cases, CT scan may be most practical, and prompt treatment with antibiotics is mandatory.

The sphenoid sinus may be the site of an infiltrative tumor (Langerhans' cell histiocytosis) associated with retro-orbital pain and frontal headache (see Headache section below for case-report in current issue of N Engl J Med Feb 14, 2002).

Spontaneous extradural hematoma with sinusitis is an unusual complication of frontal sinusitis, in a case report of a 17 year-old man treated at Princess Royal Hospital, Haywards Heath, UK (Papadopoulos MC et al. IRSM Nov 2001;94:588-589). The patient developed a sudden exacerbation of headache, with nausea and vomiting, 3 days after antibiotic treatment for frontal sinusitis. CT showed a right frontal extradural hematoma which was evacuated surgically, with recovery. Spread of infection beyond the sinus was supported by histological and radiological evidence of inflammation in contiguous bone and dura. Bleeding was explained by weakened dural vessels, secondary to infection. A dissenting opinion was expressed in a letter to the editor (Gordon AG. IRSM February 2002;95:110-111), favoring dural pneumodissection secondary to a traumatic fistula after coughing or sneezing. Gordon cites several examples of acute severe headache with traumatic fistula and extradural hematoma, secondary to barotrauma, violent nose-blowing, or violent wheezing and asthma.

Pott's puffy tumor is another unusual complication of frontal sinusitis, reported in 7 childhood cases, ages 11 to 18 years, and seen at Case Western Reserve University School of Medicine, Cleveland, OH (Babakidis NC, Cohen AR. Pediatr Neurosurg Aug 2001;35:82-89). First described by Sir Percival Pott, surgeon at St Bartholomew's Hospital, London, in 1775, "Pott's puffy tumor" is a localized swelling of the forehead, secondary to subperiosteal or epidural abscess and a complication of trauma or frontal sinusitis. Despite the advent of antibiotics, 21 cases have been reported in children in the last 20 years. Headache, fever, nasal drainage, and frontal sinus tenderness are common presenting complaints. Four of 7 cases had acute neurological complications, including aphasia, hemiparesis, obtundation, and III nerve palsy. Intracranial infections were epidural abscess in 5 patients, subdural empyema in 4 , and brain abscess in 1 . All underwent craniotomy. The scaip was edematous and purulent. Recovery followed surgery and antibiotic therapy. In adults, another cause of Pott's puffy tumor is aseptic meningeal metastatic carcinomatosis (personal observation).

\section{$\underline{\text { ATAXIAS }}$}

\section{LANGERHANS CELL HISTIOCYTOSIS AND ACUTE ATAXIA}

A 3-year-old boy who presented with an acute cerebellar ataxia and x-ray evidence of apparent chest infection, caused by massive infiltration of Langerhans histiocytes, is reported from the Department of Pediatrics, University of Catania, Italy, Northampton General Hospital, and John Radcliffe Hospital, Oxford, UK. Ataxia and slurred speech had been present for 6 weeks before admission. Brain CT and MRI were normal. Chest X-ray showed widespread reticulonodular shadowing, suggestive of mycoplasma pneumonia or metastatic disease. After 2 days treatment with erythromycin for presumed pneumonia, the 